\title{
Análisis descriptivo comparativo entre proyectos integradores de saberes en carreras afines a comunicación
}

\section{Comparative descriptive analysis between projects integrating knowledge in careers related to communication}

Lcdo. José Rafael Salguero Rosero, MSc.

Universidad Nacional de Chimborazo, Ecuador

Lcda. Tatiana del Rosario Cedillo Jurado, MSc.

Universidad Internacional del Ecuador, Ecuador

Dr. Jorge Ricardo Rodríguez Espinosa, MSc.

Universidad Pedagógica Enrique José Varona, Cuba

Autor para correspondencia: jsalguero@unach.edu.ec, tacedilloju@internacional.edu.ec.

Fecha de recepción: 01 de Febrero de 2016 - Fecha de aceptación: 25 de Febrero de 2017

Resumen: El artículo presenta un análisis documental, descriptivo y comparativo entre la metodología de los proyectos integradores de saberes (PIS), ejecutados en la carrera de Diseño Gráfico de Universidad Nacional de Chimborazo (UNACH) y en la carrera de Comunicación Social, mención Publicidad y Diseño, de la Universidad Internacional del Ecuador (UIDE) sede Guayaquil. El objetivo es fundamentar parámetros teóricos, técnicos y metodológicos que garanticen resultados óptimos en subsiguientes proyectos integradores, desde su concepción hasta la evaluación. Presenta aportes teóricos y estructurales que pueden ser aplicados en cualquier campo disciplinar o carrera. La principal conclusión a la que se llega es: el PIS es un método estratégico didáctico para potenciar la autonomía en el aprendizaje, la experimentación y el trabajo multidisciplinario. Proporciona medios y recursos para una evaluación constante, evidenciando, tanto el crecimiento individual del educando como el progreso en equipo. La socialización de los resultados contribuirá a fortalecer las competencias didácticas de los docentes en la enseñanza superior, aplicando el aprendizaje cooperativo.

Palabras clave: proyectos integradores de saberes; interdisciplinariedad; aprendizaje cooperativo; PIS; transdisciplinariedad

\begin{abstract}
The article presents a documentary, descriptive and comparative analysis between the integration project methodology of the of knowledge (WEE), executed in Graphical Design career of Chimborazo's National University (UNACH) and Social Communication career mention in Advertising and Design, of the International University of the Ecuador (UIDE) in Guayaquil. The aim is to base theoretical, technical and methodological parameters that guarantee right results in subsequent of integration projects, from his conception up to the evaluation. It presents theoretical and structural contributions that they can be applied in any discipline field or career. The principal conclusion to the one that comes near is: the WEE is a strategic didactic method to promote the autonomy in the learning, the experimentation and the multidisciplinary work. It provides means and resources for a constant evaluation, demonstrating, both the individual growth of the pupil and the progress in equipment. It provides means and resources for a constant evaluation,
\end{abstract}


demonstrating, both the individual growth of the pupil and the progress in equipment. The socialization of the results will help to strengthen the didactic competitions of the teachers in the higher education, applying the cooperative learning.

Key Words: Projects integrating knowledge; interdisciplinary; cooperative learning; PIS; transdisciplinary

\section{Introducción}

Los paradigmas, como la sociedad, están en constante cambio y actualización; el fenómeno educativo no está exento. Observando retrospectivamente se advierten cambios profundos en los enfoques didácticos y estilos docentes. Antes de los años 60, el enfoque educativo estuvo centrado en los contenidos, evidenciando un modelo tradicionalista, academicista, cuyo objetivo era acumular y reproducir información. El rol del docente era el seleccionar, comunicar, enseñar y mostrar los saberes, a estudiantes totalmente receptivos que eran adiestrados a resolver ejercicios por reiteración mecánica, provocando un aprendizaje memorístico. En los años 70, la educación se vuelca hacia un enfoque centrado en las habilidades. Surge el aprender a pensar, para desarrollar habilidades intelectuales y disciplina en la aplicación de habilidades que resuelvan problemas. El docente estimula intereses que resulten útiles para los estudiantes. Su rol es orientar el aprendizaje, seleccionando, implementando y presentando actividades que evidencien los resultados del aprendizaje. El alumno asume un rol más protagónico, es el ejecutor de las actividades planteadas. A partir de los años 80, el enfoque educativo se centra en los conocimientos, toma fuerza el "aprendizaje significativo", se busca promover la exploración y el descubrimiento por parte del estudiante. Vygotsky gana protagonismo con su teoría constructivista y Bruner con su teoría de aprendizaje por descubrimiento. El docente se convierte en mediador entre el conocimiento específico y las comprensiones individualizadas de sus estudiantes, facilita el aprendizaje, investiga procesos y reconstruye su accionar pedagógico. El alumno revisa, modifica, utiliza y transfiere lo que aprendido.

Dicha evolución es continua. Se basa en la búsqueda de mayor autonomía y responsabilidad de los estudiantes en formación. Procura mejorar la calidad educativa, hacia una formación integral de los profesionales. Propende la evolución, tanto de docentes como estudiantes líderes, que respondan de forma eficiente y eficaz a las necesidades que demanda la sociedad en la que interactúan. En la última década, la Educación Superior en el Ecuador evidencia cambios significativos. La política vigente exige garantizar la pertinencia de los profesionales en formación, en estricta armonía con los objetivos del Plan Nacional del Buen Vivir y los problemas y tensiones nacionales, zonales y territoriales donde se establecen las universidades. Para lograr esa misión, el sistema educativo superior fue replanteado desde su concepción filosófica, evidenciada hasta hace poco en el paradigma constructivista, reconstruyéndose sobre las bases del paradigma de la complejidad sistémica. Deja atrás el predominio de una visión analítica disciplinar, donde se pensaba que cruzar conocimientos entre ciencias era "contaminar". Esta nueva filosofía pretende dejar atrás el currículo lineal, donde cada docente planificaba de forma individual sus contenidos, sin tomar en cuenta las correlaciones que existen entre dos o más asignaturas. Pretende además, eliminar el trabajo individualista, tanto del docente como del estudiante, procurando desarrollar habilidades para el trabajo en equipo y aprendizaje cooperativo. 
Para comprender de mejor manera al paradigma de la Complejidad Sistémica, (Malinowsky, 2014) citando a Edgar Morín, señala siete principios que definen un pensamiento integrador, vinculante y complejo, estos son: principio sistémico, principio holográmico, principio de bucle retroactivo o retroalimentación, principio de bucle recursivo, principio de autonomía/dependencia, principio dialógico y principio de reintroducción del observador en todo conocimiento. En síntesis, Morín expone que "mediante la formulación innovadora de los conceptos: dialogía, recursividad y hologramía, se facilitará el diálogo acerca de un mismo tema, desde diferentes puntos de vista". Entonces, se concluye que el sistema educativo actual, no da cabida a la gestión individualizada del docente, tampoco a la supremacía de un campo disciplinar sobre otro; más bien provoca una convergencia de múltiples visiones disciplinares, conceptuales, metodológicas, instrumentales, para resolver un problema complejo.

Hoy, las universidades ecuatorianas están enmarcadas en el paradigma de la Complejidad Sistémica. En citado contexto, el Modelo Educativo, Pedagógico y Didáctico de la UNACH, propone su gestión académica en torno a las líneas de interdisciplinariedad, transversalidad de aprendizajes, investigación como herramienta didáctica, diálogo intercultural, trabajo colaborativo entre redes académicas nacionales e internacionales, afectividad y práctica de valores. El docente asume un rol de carácter tutorial, cumple un acompañamiento, guía, reconduce, provoca y orienta el conocimiento. Atiende problemas reales en un contexto determinado. Mientras, el estudiante fortalece su habilidad de trabajo autónomo, sentido crítico, reflexivo, capacidad analítica y emprendedora, utilizando herramientas de investigación. Como se evidencia, tanto el docente como el estudiante requieren trabajar en equipo. No necesariamente con estudiantes de su propia carrera y centro universitario, sino que pueden orientarse a un trabajo que involucre dos o más carreras y/o dos o más universidades, siempre que existan las facilidades para su interacción y que los objetivos sean suficientemente abarcadores.

El Consejo de Educación Superior, en el Reglamento de Régimen Académico que rige a las universidades del Ecuador, en su artículo 15 literales b y c, dictamina que el componente de docencia está definido por el desarrollo de ambientes de aprendizaje, que deben incorporar actividades pedagógicas orientadas a la sistematización del conocimiento científico. Las instituciones de educación superior, considerando la citada reglamentación, determinaron como eje metodológico didáctico el desarrollo de proyectos integradores de saberes (PIS), cuya finalidad es fomentar un trabajo interdisciplinario y transdisciplinario, que se ajusta al paradigma vigente de la complejidad sistémica. De una revisión a los trabajos expuestos en los repositorios digitales de las universidades ecuatorianas, que abordan temáticas afines a PIS, se puede extraer las siguientes convergencias teórico-metodológicas: el PIS es la integración y no la suma de partes. Para la ejecución de los PIS se requiere un aporte sistemático de las asignaturas, de un nivel, un campo disciplinar o un constructo académico. El PIS construye vinculaciones interdisciplinarias entre las asignaturas que se integran. El origen del PIS está en problemas reales en un contexto real al que tienen acceso los participantes. Las estrategias básicas para desarrollar PIS son: el trabajo en equipo, el aprendizaje cooperativo y la aplicación de las habilidades investigativas. 
Si bien la fundamentación teórica sobre PIS es explícita, la complejidad se presenta cuando se inicia el desarrollo de los proyectos. El accionar docente puede conducir por múltiples metodologías, en procura de ejecutar el proyecto ajustado a los periodos académicos vigentes en cada institución, a las necesidades de cada contexto, a los objetivos de aprendizaje planteados en las asignaturas convergentes en el PIS y a las políticas de vinculación con la sociedad, a las que responden las universidades. Otro reto es el aspecto operativo, determinar el docente que liderará el PIS, asignar actividades a los docentes participantes, establecer un cronograma colectivo que debe ser respetado, determinar tareas específicas para cada asignatura, organizar los equipos de trabajo con los estudiantes, reglamentar los procesos de guía, tutoría y determinar las rúbricas de heteroevaluación y coevaluación, tanto a docentes como a estudiantes.

El objetivo del artículo es fundamentar parámetros teóricos, técnicos y metodológicos que garanticen resultados óptimos en subsiguientes proyectos integradores, desde su concepción hasta la evaluación. Presenta aportes teóricos y estructurales que pueden ser aplicados en cualquier campo disciplinar o carrera. La principal conclusión a la que se llega es: el PIS es un método estratégico didáctico para potenciar la autonomía en el aprendizaje, la experimentación y el trabajo multidisciplinario. Proporciona medios y recursos para una evaluación constante, evidenciando, tanto el crecimiento individual del educando como el progreso en equipo. La socialización de los resultados contribuirá a fortalecer las competencias didácticas de los docentes en la enseñanza superior, aplicando el aprendizaje cooperativo.

\section{Material y métodos}

La investigación efectuada utiliza el método analítico descriptivo. Pretende alcanzar, a través de la revisión de documentos teóricos, la observación directa e indirecta y la comparación entre PIS efectuados en dos instituciones superiores, una sustentación teórica metodológica para el desarrollo de PIS desde su concepción hasta su evaluación. El aporte significativo gira en torno a lo procedimental, ya que describe paso a paso las acciones necesarias para concretar un PIS. Detalla la determinación de las asignaturas convergentes, los núcleos problémicos pertinentes, el puente integrador, la construcción de la meta-competencia que desarrollará el PIS, el desarrollo de las actividades sistemáticamente organizadas, la construcción del cronograma de actividades, las orientaciones para docentes y estudiantes en cada fase y los procedimientos de evaluación.

En cuanto a la muestra o unidades de análisis se determinaron dos: el PIS denominado "Manual de identidad para una empresa, producto, servicio o territorio, sustentado en un proceso de investigación científica" realizado por los estudiantes de primer y segundo semestre de la carrera de Diseño Gráfico de la UNACH, bajo la dirección del docente Rafael Salguero y el PIS denominado "El día de Simón, exposición de propuestas culturales" realizado por los estudiantes de la carrera de Comunicación Social, mención Publicidad y Diseño Gráfico de la UIDE, bajo la tutoría de Tatiana Cedillo. Los dos PIS se desarrollaron simultáneamente, desde su proceso de concepción hasta su presentación y evaluación. Los proyectos se seleccionaron por correlación temática, aproximación de competencias, y afinidad entre investigadores.

En la fase analítica, cada proponente analizó los perfiles de proyectos integradores, donde se detallan: las asignaturas convergentes, los objetivos de la carrera equiparados a los objetivos 
del Plan Nacional del Buen Vivir, los resultados de aprendizaje por cada asignatura, las competencias por niveles y los reglamentos de evaluación en cada institución. Adicionalmente, se observó el perfil de los docentes participantes, para la asignación de las actividades según sus competencias y talentos.

Las técnicas de levantamiento de información fueron: la entrevista, con los docentes participantes y con los líderes o representantes de los campos de investigación donde se insertaron, la observación directa, a los sílabos de las asignaturas, el grupo focal, para la consolidación de los perfiles de PIS. Además en cada proyecto se utilizaron técnicas e instrumentos particulares como: encuesta índice de Anholt, pauta etnográfica, encuesta, entre otros. La información particular de cada PIS se presentó a través de tablas y gráficas estadísticas, así también con análisis descriptivos y pizarras gráficas. Como criterios de selección de información, algunas particularidades se describirán en la fase de desarrollo y otras se pueden observar en los anexos.

Vale añadir que los PIS desarrollados se ajustaron a las normativas y reglamentaciones de cada universidad. Para la revisión de la documentación, se contó con la aprobación de los representantes de los campos de estudio. Los instrumentos de investigación se aplicaron con consentimiento informado de parte de todos los sujetos que intervinieron en la ejecución de los PIS.

\section{Resultados}

El estudio se basó en el análisis de los sílabos correspondientes a las asignaturas participantes. Para el caso de la UNACH: Metodología de Investigación, Diseño Gráfico I, Fundamentos de Diseño, Expresión Gráfica y Computación Gráfica I. Para el caso de la UIDE se consideraron los 4 ejes de la carrera: 1) Investigación y Redacción. 2) Arte, Diseño y Creatividad. 3) Comunicación Organizacional, mediática y manejo de audiencias. 4) Audiovisual y Multimedia. Las unidades de estudio fueron: asignaturas convergentes, objetivos de las asignaturas y/o ejes y núcleos problémicos.

Cumplido el análisis comparativo de los dos perfiles de PIS de las universidades y carreras citadas, se presentan los resultados teóricos, estructurales y metodológicos evidenciados en uno u otro PIS, que permiten proponer un orden sistemático así:

1. Para iniciar con el PIS se requiere determinar la integración curricular por semestres, nivel de organización (básica, profesional, titulación) o campo disciplinar, tal como se muestra en la gráfica 1 . 


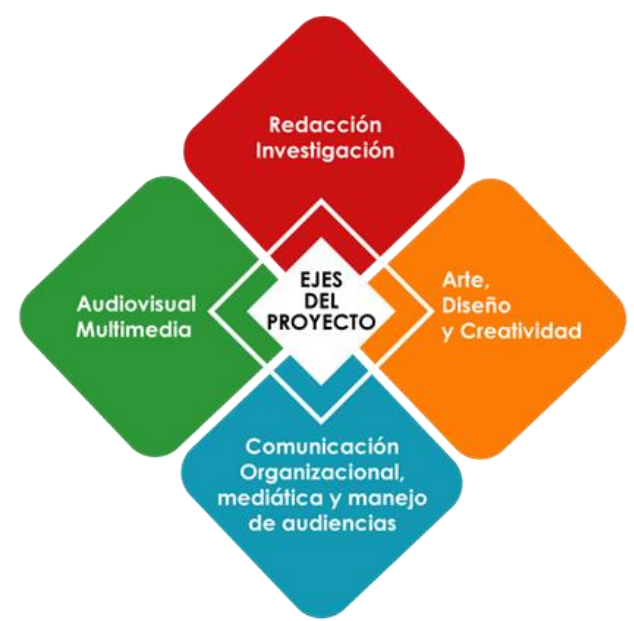

Gráfica 1.- Ejes integrados en el PIS de la UIDE

Fuente: Cedillo, T. (2016) Proyecto Integrador de Saberes UIDE

2. Una vez determinadas las asignaturas convergentes se procedió a enlistar los núcleos problemáticos de cada una (noción conceptual macro, concepto no directamente incluido en el listado de temas de la asignatura, pero que emerge de su estructura general). Ver tabla 1 .

Tabla 1: Asignaturas, objetivos y núcleos problémicos considerados/UNACH.

\begin{tabular}{|c|c|c|}
\hline Asignatura & Objetivos & Núcleos problémicos \\
\hline $\begin{array}{l}\text { Métodos de } \\
\text { Investigación }\end{array}$ & $\begin{array}{l}\text { Demostrar habilidades de trabajo autónomo, uso de } \\
\text { técnicas de estudio y aplicación del método científico } \\
\text { para la interpretación de la realidad. }\end{array}$ & $\begin{array}{l}\text { Técnicas de estudio, } \\
\text { Métodos de } \\
\text { Investigación. }\end{array}$ \\
\hline Diseño Gráfico I & $\begin{array}{l}\text { Aportar con la base teórica y práctica para la gestión y } \\
\text { construcción de un identificador visual, mismo que } \\
\text { impulsa el desarrollo de una empresa, producto, servicio } \\
\text { o territorio, mediante su función identificadora para su } \\
\text { inmersión en el mercado, incrementando la capacidad } \\
\text { del estudiante en buscar soluciones de problemas de } \\
\text { índole visual. }\end{array}$ & $\begin{array}{l}\text { Identificador Visual, } \\
\text { investigación de } \\
\text { mercado, problemas de } \\
\text { comunicación visual, } \\
\text { marcas. }\end{array}$ \\
\hline $\begin{array}{l}\text { Fundamentos } \\
\text { del Diseño }\end{array}$ & $\begin{array}{l}\text { Reconocer y analizar la importancia de los mensajes } \\
\text { visuales, para plasmar sus ideas con un fundamento } \\
\text { teórico sustentable. }\end{array}$ & $\begin{array}{l}\text { Mensajes visuales, } \\
\text { deconstrucción de } \\
\text { comunicación visual, } \\
\text { composición gráfica. }\end{array}$ \\
\hline $\begin{array}{l}\text { Expresión } \\
\text { Gráfica II }\end{array}$ & $\begin{array}{l}\text { Dominar recursos y técnicas manuales de expresión, } \\
\text { enfocadas a propuestas de Diseño Gráfico, con criterio } \\
\text { estético y funcional, contribuyendo al desarrollo social y } \\
\text { al arte en el entorno al que pertenece. }\end{array}$ & $\begin{array}{l}\text { Estética, expresión } \\
\text { gráfica, mensajes } \\
\text { visuales, caracterización } \\
\text { de públicos objetivos. }\end{array}$ \\
\hline $\begin{array}{l}\text { Computación } \\
\text { Gráfica I }\end{array}$ & $\begin{array}{l}\text { Desarrollar propuestas creativas y funcionales mediante } \\
\text { el software de ilustración para su aporte a la producción } \\
\text { gráfica. }\end{array}$ & $\begin{array}{l}\text { Imágenes vectoriales, } \\
\text { ilustración digital, } \\
\text { producción gráfica. }\end{array}$ \\
\hline $\begin{array}{l}\text { Realidad } \\
\text { Nacional y } \\
\text { Gobernabili-dad }\end{array}$ & $\begin{array}{l}\text { Integra saberes de índole investigativo y educativo, } \\
\text { desde la complejidad, mediante actividades de carácter } \\
\text { teórico-metodológico y práctico de acuerdo con los } \\
\text { requerimientos de índole disciplinar y profesional, } \\
\text { conformando equipos de trabajo. }\end{array}$ & $\begin{array}{l}\text { Investigación local, } \\
\text { gobernabilidad, saberes } \\
\text { ancestrales. }\end{array}$ \\
\hline
\end{tabular}

Fuente: Salguero, R. (2016) Proyecto Integrador de Saberes - UNACH 
Tabla 2: Asignaturas, objetivos y núcleos problémicos considerados/UIDE.

\begin{tabular}{|c|c|c|}
\hline Ejes (asignaturas) & Objetivos & Núcleos problémicos \\
\hline $\begin{array}{l}\text { Eje } 1 . \\
\text { Investigación y redacción } \\
\text { Asignaturas } \\
\begin{aligned} \text { 1. } & \text { Composición } \\
2 . & \text { Historia del Periodismo } \\
\text { 3. } & \text { Redacción y Estilo } \\
4 . & \text { Investigación cuantitativa y } \\
& \text { cualitativa }\end{aligned}\end{array}$ & $\begin{array}{l}\text { Desarrollar habilidades de } \\
\text { conceptualizar un tema, organizar la } \\
\text { información, preparar datos que } \\
\text { evidencien una investigación } \\
\text { minuciosa en el manejo de contenido, } \\
\text { gestionar la planificación para } \\
\text { difusión. }\end{array}$ & $\begin{array}{ll}\text { - } & \begin{array}{l}\text { Estructura para } \\
\text { desarrollo de } \\
\text { proyectos. }\end{array} \\
\text { - } & \text { Manejo de sintaxis y } \\
\text { ortografía. } \\
\text { - Manejo de } \\
\text { información para } \\
\text { conceptualización de } \\
\text { temas }\end{array}$ \\
\hline 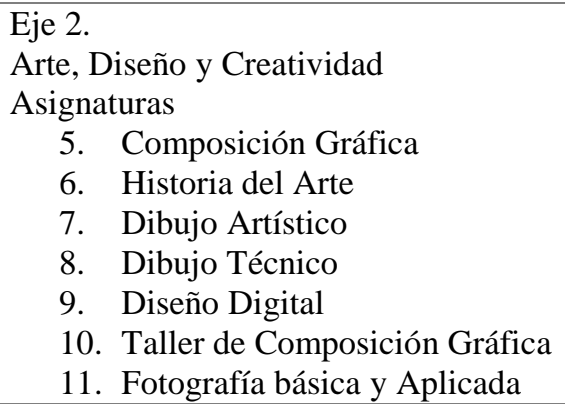 & $\begin{array}{l}\text { Analizar y reflexionar sobre el diseño } \\
\text { gráfico y su relación con la } \\
\text { publicidad, impulsando a colaborar } \\
\text { con el material recopilado de la } \\
\text { investigación previa para la correcta } \\
\text { elaboración de piezas gráficas. }\end{array}$ & $\begin{array}{l}\text {-Criterio de selección } \\
\text { fotográfica } \\
\text {-Composición de páginas } \\
\text { (diagramación) } \\
\text {-Campañas publicitarias } \\
\text { empleando el uso de } \\
\text { herramientas necesarias. }\end{array}$ \\
\hline $\begin{array}{l}\text { Eje } 3 . \\
\text { Comunicación Organizacional, } \\
\text { mediática y manejo de audiencias } \\
\text { Asignaturas } \\
\text { 12. Introducción a la } \\
\text { Comunicación } \\
\text { 13. Medios de Comunicación } \\
\text { 14. Sociología de la Comunicación } \\
\text { 15. Comunicación Organizacional, } \\
\text { RR.PP. } \\
\text { 16. Comunicación Corporativa. }\end{array}$ & $\begin{array}{l}\text { Emplear habilidades de producción } \\
\text { de contenidos, circulación y consumo } \\
\text { de significados entre una } \\
\text { organización y sus públicos. }\end{array}$ & $\begin{array}{l}\text {-Gestión de estrategias } \\
\text { comunicacionales y de } \\
\text { promoción. } \\
\text {-Detección de problemas de } \\
\text { comunicación de manera } \\
\text { anticipada } \\
\text {-Planificación, conducción y } \\
\text { organización de actividades. }\end{array}$ \\
\hline $\begin{array}{l}\text { Eje } 4 . \\
\text { Audiovisual - Multimedia } \\
\text { Asignaturas } \\
\quad \text { 17. Multimedia Design }\end{array}$ & $\begin{array}{l}\text { Aplicar técnicas de optimización de } \\
\text { contenidos multimedia para la } \\
\text { correcta difusión de contenidos en la } \\
\text { Web. }\end{array}$ & $\begin{array}{l}\text {-Manejo del sistema } \\
\text { multimedia. } \\
\text {-Manejo de redes. }\end{array}$ \\
\hline
\end{tabular}

Fuente: Cedillo, T. (2016) Proyecto Integrador de Saberes - UIDE

3. Posteriormente se trabajó en una matriz de reintegración de los núcleos problémicos, considerando el número de asignaturas en las que está presente dicho núcleo. El núcleo que esté presente en el mayor número de asignaturas se constituirá en el "puente integrador", tal como se muestra en las tablas 3 y 4

Tabla 3.- Matriz de reintegración de núcleos problémicos / UNACH

\begin{tabular}{ll}
\hline Puente Integrador & $\mathbf{N}^{\mathbf{o}}$ Asignaturas \\
\hline Proceso de Investigación & $1,2,6$ \\
\hline Identificador Visual & $1,3,5$ \\
\hline Mensaje Visual & $2,3,4,5,7$ \\
\hline
\end{tabular}

Fuente: Salguero, R. (2016) Proyecto Integrador de Saberes/UNACH 
Tabla 4.- Matriz de reintegración de núcleos problémicos / UIDE

\begin{tabular}{ccc}
\hline Puente Integrador & \multicolumn{1}{c}{$\mathbf{N}^{\mathbf{o}}$ Asignaturas } \\
\hline Conceptualización de temas & - & $1,2,3,4,5,6,9,10$ \\
\hline Composición de páginas y diseño & - & $5,7,8,9,10,11$ \\
\hline Estrategias comunicacionales y de promoción & - & $10,12,13,14,15,16$ \\
\hline Aplicación de sistemas multimedia & - & 17 \\
\hline
\end{tabular}

Fuente: Cedillo, T. (2016) Proyecto Integrador de Saberes/UIDE

La integración de los contenidos de las asignaturas evidencia los puentes integradores que comparten una misma conexión, lo que justifica y sustenta la colaboración entre los docentes involucrados por de las asignaturas involucradas en el PIS, garantizando que los contenidos son convergentes. Vea gráfico 2.

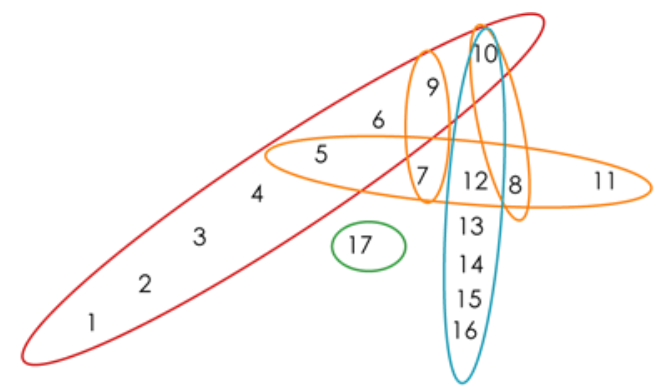

Gráfica 2.- Intersección de núcleos problémicos entre asignaturas del PIS/UIDE Fuente: Cedillo, T. (2016) Proyecto Integrador de Saberes/UIDE

4. Identificación de las macro-competencias y acciones didácticas.- Una vez acordado el puente integrador entre las asignaturas convergentes, se procedió a enlistar las competencias por cada asignatura. En tal sentido, se presentaron algunos casos de sílabos donde no se encontraban detalladas dichas competencias, por lo que se procedió a describirla en base al objetivo de la asignatura, considerando que, una competencia debe evidenciar al menos, los saberes teóricos, prácticos y axiológicos. En base a las competencias específicas se redactó la macro-competencia que desarrollarán los estudiantes, con la ejecución del PIS propuesto. Además se describieron las acciones didácticas que cada docente involucrado debió cumplir dentro del PIS. Los resultados del trabajo efectuado en este aspecto pueden ser apreciados en la tabla 5. 


\section{Tabla 5.- Planeación de acciones didácticas en base al puente integrador/PIS UNACH}

\section{PUENTE INTEGRADOR}

$\begin{array}{ll}\text { Mensaje Visual } & \begin{array}{l}\text { Métodos de } \\ \text { Investigación }\end{array}\end{array}$

Diseño Gráfico I

Fundamentos del

Diseño

\section{Expresión}

Gráfica II

Computación

Gráfica I

\section{MICRO Y MACROCOMPETENCIAS}

- Desarrolla el proceso de investigación en una temática determinada y lo evidencia mediante la presentación y sustentación del análisis e interpretación de resultados.

- Integra saberes de índole investigativo y educativo, desde la complejidad, mediante actividades de carácter teórico-metodológico y práctico de acuerdo con los requerimientos de índole disciplinar y profesional, conformando equipos de trabajo.

- Construye y gestiona identificadores visuales de una empresa, producto, servicio o territorio.

- Utiliza la composición de formas, estructuras y los principales fundamentos del Diseño.

- Domina recursos y técnicas manuales de expresión enfocadas a propuestas de Diseño Gráfico.

- Comprende y aplica conocimientos teórico/prácticos para la elaboración de propuestas en base a imágenes vectoriales.

\section{ACTIVIDADES/ACCIONES DIDÁCTICAS}

- Fundamentación de los componentes del proceso de investigación

- Elaboración de los instrumentos para gestionar información bibliográfica y producto de la interacción con las personas. (Contemplar: Lluvia de ideas, Benchmarking, Pauta Etnográfica, Pizarras Gráficas)

- Utilización de técnicas para el procesamiento y análisis de los resultados de la aplicación de instrumentos.

- Determinar la empresa, producto, servicio o territorio local, objetos de investigación.

- Establecer el proceso de gestión de la marca.

- Determinar la tipología de marca más adecuada para el Estudio de Caso.

- Supervisar el proceso creativo

- Generar bocetos aplicando los fundamentos del Diseño.

- Diversificar propuestas mediante la aplicación del enfoque creativo.

- Aplicar el proceso cromático mediante técnicas secas y húmedas.

- Presentar bocetos concluidos a color

- Desarrollar el proceso de redibujo de los bocetos ganadores.

- Guiar el proceso de maquetación del Manual de Identidad de Marca.

\section{$\checkmark$ RESULTADO DE APRENDIZAJE}


- Construye y gestiona identificadores visuales de una empresa, producto, servicio o territorio local, sustentado en instrumentos y métodos de investigación científica, evidenciadas en aplicaciones de técnicas manuales y digitales.

\section{- PROBLEMA DE INVESTIGACIÓN}

- ¿Cómo mejorar o proponer una nueva marca en una empresa, producto, servicio o territorio local,

basada en una investigación científica?
Diseñar el manual de identidad de una empresa, producto, servicio o territorio local, sustentado en instrumentos y métodos de investigación científica, evidenciadas en aplicaciones de técnicas manuales y digitales.

Fuente: Salguero, R. (2016) Proyecto Integrador de Saberes/UNACH 
Como se observa en la Tabla $N^{\circ}$ 5, bajo la meta-competencia se detalla además el problema de investigación. Es importante dicho ítem porque todo PIS debe responder a una problemática real de un contexto específico. Es aquí donde el colectivo de docentes debe definir el campo de investigación, las técnicas e instrumentos a aplicar; además de garantizar el acceso a la información, tanto a nivel bibliográfico conceptual, como a los datos estadísticos cuantitativos y datos cualitativos que demande el PIS.

5. Desarrollo.- Esta fase es fundamental y está asociada con la ejecución del PIS. La conforman las acciones didácticas que se detallan en la columna derecha de la tabla $\mathrm{N}^{\circ} 5$. respecto al desarrollo, Cabe mencionar que de preferencia asumirá el liderazgo del PIS, el docente que tenga mayor pertinencia con el núcleo problémico integrador, que comandará y guiará las acciones didácticas para desarrollar competencias propias de la profesión y por ende, su perfil profesional es el más acorde para cumplir dicha función. Cada docente participante conocerá, desde el inicio, las acciones o actividades y los tiempos en los que debe concretar sus aportes. El PIS se puede comparar con una carrera de "postas". El éxito depende de la sinergia entre docentes y estudiantes, si uno u otro incumple y no entrega su "testigo" a tiempo, retrasará todo el proceso.

En el desarrollo se contempló las siguientes puntualizaciones:

- La propuesta del proyecto debe redactarse en términos claros y concisos, el alcance debe estar de acuerdo al nivel de formación en el que se encuentran los estudiantes.

- Se debe formular objetivos del proyecto, se citó un objetivo específico por asignatura integrada.

- Los docentes deben garantizar la socialización de información conceptual y metodológica, que contribuya a comprender las variables de investigación propuestas.

- Si la propuesta amerita, se puede formular una hipótesis o preguntas científicas, en dependencia con el paradigma que cobije el proceso y del nivel que cursen los estudiantes. Esto aporta a delimitar el campo de acción del trabajo propuesto.

- Se requiere determinar las técnicas e instrumentos de recolección de datos y supervisar el ejercicio de dichas tareas.

- Se debe acordar colectivamente la planificación de actividades propuestas, que garanticen la consecución del PIS.

- Se requiere determinar los espacios, tiempos y recursos para la ejecución; estos lineamientos se definen en la fase de planificación y evidencian en el perfil del PIS.

6. Evaluación.- Docentes y estudiantes que integran el PIS deben conocer que la evaluación es continua. Inicia desde la fase de planificación, prosigue en cada actividad o acción didáctica propuesta, hasta la fase de evaluación final. Se debe considerar que, no solo los estudiantes serán los evaluados, sino también el líder del proyecto y los docentes que integran el equipo de trabajo. Además, se debe considerar indicadores de evaluación que no midan solo conocimientos teóricos y habilidades prácticas evidenciadas; también deben considerarse criterios procedimentales, actitudinales y axiológicos, considerando la integralidad de la formación universitaria. Las rúbricas de evaluación deben ser socializadas en la fase de planificación, para que, tanto docente como estudiantes conozcan los indicadores que incidirán en su nota final. Cada docente está en libertad de 
utilizar sus propias rúbricas en las acciones didácticas que le competen individualmente, pero, es necesario socializar una rúbrica global con la cual se evaluarán los resultados finales. Ver anexo/rúbricas propuestas.

Una vez ejecutados los dos PIS, también se puede evidenciar como resultado la construcción del perfil deseado del docente líder. Citado perfil surge de un análisis crítico reflexivo entre los autores del presente artículo.

El líder del Proyecto Integrador de Saberes debe:

- Contar con el perfil profesional acorde a la carrera, al campo de conocimiento y/o al objeto de estudio que abordará el PIS.

- Poseer cualidades y aptitudes de liderazgo cooperativo.

- Tener dominio de los roles y funciones del trabajo en equipo.

- Demostrar habilidades: teóricas, prácticas y metodológicas, afines a los problemas de investigación propios de cada carrera.

- Asumir poder de convocatoria para desarrollar, cuando sea necesario, talleres y clases colectivas entre los docentes participantes en el PIS.

- Tener dominio de metodologías para trabajo en equipo, así también sobre metodologías propias de la profesión.

- Tener dominio para el diseño, evaluación y aplicación de rúbricas de evaluación.

- Ser puntual, colaborativo, solidario, exigente y disciplinado.

- Contar con habilidades comunicativas para suscribir acuerdos de vinculación entre la carrera y los actores y sectores que demandan los servicios profesionales.

- Tener vocación y buena voluntad para comprometerse con el proyecto planteado.

Vale destacar que, no siempre es necesario que el líder sea un docente de especialidad, cuando existe la voluntad y la vocación, cualquier docente puede asumir el rol de líder, considerando que tendrá un equipo multidisciplinario que lo acompañe y un equipo de estudiantes que están predispuestos a trabajar bajo su direccionamiento.

\section{Discusión de resultados}

Al término del análisis comparativo entre los dos PIS, UNACH/UIDE, se determinó que la principal diferencia corresponde al número de asignaturas convergentes contempladas. En el caso de la UNACH se vincularon a un inicio seis asignaturas, posteriormente se desvinculó una, Realidad Nacional y Gobernabilidad. Para el caso de la UIDE se vincularon cuatro ejes con un total de 17 asignaturas. No se podría establecer un número ideal de asignaturas, todo dependerá del liderazgo y la planificación existente, pero si se puede citar que, a mayor número de asignaturas, docentes y estudiantes participantes, mayor exigencia en la planificación.

En cuanto a los aspectos organizativos es importante citar que para la ejecución de las acciones didácticas, sobre el docente participante recae la principal responsabilidad. De ser necesario, se establecerá equipos de trabajo entre estudiantes, con un líder por equipo, que asuma la responsabilidad de facilitar o guiar los procesos en las actividades de trabajo autónomo, 
aplicación o experimentación, cuando estas se cumplan fuera de las aulas y en horas extra-clase. Como aspecto positivo se cita que en ambos PIS, se contó con la participación y cooperación activa, tanto de docentes como de estudiantes. En cuanto a la organización se resalta la importancia del trabajo en equipo en todas las etapas, la socialización entre todos los actores que intervendrán en el PIS, antes de iniciar el proceso, la supervisión continua en todas las acciones didácticas y la evaluación bajo parámetros detallados con anterioridad.

La planificación de micro-talleres, así como clases compartidas entre dos o tres docentes, dependiendo los contenidos teóricos contemplados, se hizo necesaria. La necesidad surge cuando los contenidos teóricos deben ser direccionados a la aplicación en acciones prácticas, más aún en los aspectos metodológicos o de investigación. Para coordinar acciones como estas, que no están contempladas en la planificación, el líder del PIS debe estar atento a los requerimientos de sus docentes participantes y motivar el trabajo colaborativo, tanto en aspectos teóricos, prácticos, metodológicos, como también en aspectos organizativos, de tutoría, e incluso en aspectos logísticos (espacios, recursos tecnológicos, entre otras necesidades)

Para cada PIS se requiere un cúmulo de información bibliográfica, si bien cada docente cuenta con el detalle de bibliografía en sus sílabos, el líder del PIS debe considerar si la información detallada es coherente y suficiente para el cumplimiento de los objetivos planteados. De no ser el caso, deberá direccionar hacia las bases de datos existentes, sea en bibliotecas físicas o repositorios digitales. Cuando el PIS requiera información particular de una empresa u organización pública o privada, el líder, o cualquier integrante garantizará el acceso a dicha información. De ser necesario se suscribirá acuerdos de confidencialidad del manejo de la información. En cuanto al aspecto metodológico es importante que cada PIS proponga una metodología global que debe ser socializada entre docentes y estudiantes, esto no limita a que los docentes apliquen en sus clases particulares otras metodologías, pero si contribuye a que el desarrollo del PIS esté bien encaminado. La metodología deberá ser consensuada entre los docentes participantes; tendrán prioridad las metodologías propias de la profesión.

Para la ejecución del PIS, en la primera fase resaltan las tareas de investigación exploratoria y descriptiva. En la mayoría de casos es necesario desarrollar instrumentos para la recopilación de datos. Considerando que los estudiantes acudirán al campo de investigación a levantar una línea base, es necesario que en el proceso de confección de los instrumentos exista una supervisión permanente y una validación de al menos dos docentes participantes. Se debe garantizar que estos instrumentos estén bien redactados, no contengan errores ortográficos y lo más importante, no tengan ni falta ni exceso de ítems, garantizando así la fiabilidad de la información recopilada.

Es enriquecedor que cada equipo de trabajo recopile evidencias del trabajo en cada una de las fases y acciones didácticas. El líder debe considerar que las actividades propuestas a cada equipo se ajusten al nivel en el cual están los estudiantes. No se pueden asignar tareas para las cuales no están preparados, peor aún sin una supervisión. Durante las actividades de docencia, los profesores deben direccionar los contenidos teóricos hacia los objetivos del PIS. En el caso de la UNACH, este direccionamiento incidió en la modificación del orden de temas contemplados en el sílabo. Se debe tener presente que las actividades son secuenciales y el avance del proyecto depende del cumplimiento de cada participante. Cabe destacar que es 
enriquecedor que los PIS permitan interactuar a estudiantes de distintos niveles, esto contribuye a que los estudiantes de niveles o semestres superiores aporten con sus experiencias a estudiantes de niveles inferiores Estos contribuyen con sus observaciones en un proceso evaluativo a sus pares y los motivan, al presenciar las capacidades que pronto adquirirán en su proceso formativo.

Dicha experiencia fue más enriquecedora en el PIS de la UIDE, donde intervinieron estudiantes de todos los niveles.

El proyecto integrador de saberes evidencia la actividad cognoscitiva del estudiante, dando significado al contenido de las asignaturas de uno o varios niveles, para solucionar un problema existente en un contexto real. El PIS compila el conocimiento teórico y las habilidades prácticas que acreditan el progreso de lo aprendido. Esa práctica educativa vivenciada, posibilita nuevas interrogantes y maximiza la exploración hacia nuevos problemas. No solo es un proyecto de aula, es la evidencia del pensamiento estratégico que conlleva un proceso de concepción, desarrollo y evaluación. Evidencia las habilidades de investigación para proponer soluciones a problemas articulados a las asignaturas competentes. El PIS, genera una motivación explícita del trabajo en equipo, no solo entre alumnos, sino que origina una sinergia entre docentes y evidencia el trabajo interdisciplinario. Esto exige perfiles de liderazgo, habilidades para asignar roles y funciones entre los integrantes; requiere de una planificación, un procesamiento y ordenamiento de datos, una redacción crítica-reflexiva de los resultados de investigación y una presentación formal que servirá para la fase evaluativa del proceso formativo.

Se debe diferenciar entre convergencia de conocimientos y convergencia de docentes. Se puede presentar el caso donde, las asignaturas entre uno o más niveles tienen núcleos problémicos que convergen, pero que la convergencia sea débil entre los docentes que dictan citadas cátedras. El líder debe ser audaz para sortear esas debilidades, proponiendo acciones que contribuyan a obviar las diferencias posibles entre los docentes, en búsqueda de un objetivo colectivo enriquecedor desde las diferencias.

El proyecto integrador de saberes constituye un proceso investigativo formativo. En todas sus fases se generó aportes teóricos, metodológicos y prácticos, que contribuyeron a fortalecer las competencias profesionales del estudiante, así también del docente. Como un ejercicio añadido, los PIS dotan de material para la redacción de ponencias, artículos de revisión o artículos de investigación que deberían socializarse en talleres, conferencias, congresos o revistas. La socialización de los resultados contribuirá a fortalecer la ejecución de PIS, además dotará de importante material instrumental que puede ser aplicado en diferentes contextos.

\section{Conclusión}

El análisis comparativo metodológico entre los PIS ejecutados en la UNACH y la UIDE contribuyó a detectar errores y aciertos. Se concluye que la fase esencial del PIS es la planificación, y dentro de esta fase, el punto fundamental es encontrar el puente integrador entre las asignaturas convergentes. Determinar este puente garantiza formular el problema, campo de intervención y objetivos del proyecto, sobre lo cual se construyen las acciones didácticas que garantizarán su cumplimiento. 
La ejecución de los PIS garantiza de manera plena la motivación continua del estudiante en su proceso de aprendizaje, e influye en la resolución de problemas a nivel de equipos de trabajo, dejando atrás el individualismo. El PIS logra ser un método estratégico didáctico para potenciar la autonomía en el aprendizaje, la experimentación y el compromiso en trabajos multidisciplinarios que evidencian el uso de herramientas que proporciona cada asignatura. El PIS proporciona medios y recursos para una evaluación constante, lo que permite evidenciar el crecimiento del educando de manera continua. La rúbrica de evaluación, por tanto, pasa a ser un elemento indispensable para el desarrollo de los PIS, el estudiante recibe una retroalimentación conforme el proyecto avanza, desde la fase de planificación, hasta la puesta en escena. La flexibilidad es punto importante dependiendo de la entidad educativa, cada una maneja de manera diferente los proyectos, pero todas llegan al mismo fin: trabajar en equipo con una metodología establecida para el logro de objetivos, por medio de la aplicación y experimentación de los aprendizajes, bajo una teoría fundamentada.

El PIS exige la manifestación de líderes, tanto a nivel de docentes como estudiantes. Esa exigencia contribuye a desarrollar habilidades comunicativas, de trabajo y aprendizaje cooperativo, habilidades de negociación, además de evidenciar posibles alternativas de emprendimientos. Entre las acciones didácticas se detallan algunas que motivan la interacción directa con el futuro campo profesional de los estudiantes en formación, evidencia además ciertas falencias del proceso académico que requiere inmediatas alternativas de solución. El PIS es un "proceso vivo" que eleva las exigencias tanto a docentes como a estudiantes, genera escenarios de aprendizaje fuera del aula, repercute en las relaciones interpersonales entre el docente, el estudiante, el que oferta plazas laborales y el entorno laboral. Es una concepción sistémica y compleja que transforma todo el proceso de enseñanza aprendizaje, incidiendo positivamente en la mejora de la calidad educativa.

\section{Bibliografía}

Aranda, A. (2014) Reglamento de Régimen Académico del Consejo de Educación Superior y estrategias de Diseño Curricular. Presentaciones ppt del Taller de Rediseño Curricular de AFEFCE. Ambato.

Cedillo, T. (2016) Proyecto Integrador de Saberes El Día de Simón. Universidad Internacional del Ecuador-sede Guayaquil.

González, A. (2015) El diseño teórico y metodológico de la investigación científica. Presentaciones ppt del Taller de Metodología de la Investigación, UNACH. Riobamba.

Fernández, A. (2016) Roles y funciones para el trabajo en equipo. Presentaciones ppt del Taller Metodologías Activas de Aprendizaje, UNACH. Riobamba.

Guffante, T. (2015) Proyectos Integradores de Saberes. Presentación ppt del Taller de Proyectos Integradores de Saberes. Universidad Nacional de Chimborazo. Riobamba.

Larrea de Granados, E. (2014) Reglamento de Régimen Académico del Consejo de Educación Superior. Quito. 
Loza, C., Guffante, T. y Malinowsky, N. (2014) Modelo educativo, pedagógico y didáctico Aproximación epistemológico-metodológica, desde la Complejidad, para el desarrollo integral de la persona, rearticulando la investigación, formación y vinculación. Universidad Nacional de Chimborazo. Riobamba.

Morín, E. en Loza, C., Guffante, T. y Malinowsky, N. (2014) Modelo educativo, pedagógico y didáctico Aproximación epistemológico-metodológica, desde la Complejidad, para el desarrollo integral de la persona, rearticulando la investigación, formación y vinculación. Universidad Nacional de Chimborazo. Riobamba.

Ramírez, H. (2007) El proyecto integrador: una estrategia pedagógica para lograr la integración y la socialización del conocimiento. Psychología. Avances de la disciplina, vol.1. $\mathrm{n}^{\mathrm{o}} 1$. Bogotá, Colombia.

Salguero, R. (2016) Proyecto Integrador de Saberes Manual de identidad para una empresa, producto, servicio o territorio, sustentado en un proceso de investigación científica. Universidad Nacional de Chimborazo. Riobamba.

Salguero, R. (2016) Rediseño curricular de la carrera de Diseño Gráfico. Universidad Nacional de Chimborazo. Riobamba.

Vygotsky L., Piaget, J. y Bruner, J. en Loza, C., Guffante, T. y Malinowsky, N. (2014) Modelo educativo, pedagógico y didáctico Aproximación epistemológico-metodológica, desde la Complejidad, para el desarrollo integral de la persona, rearticulando la investigación, formación y vinculación. Universidad Nacional de Chimborazo. Riobamba.

\section{Agradecimientos}

Es meritorio reconocer a quienes integraron los PIS en las dos universidades, campo de estudio del trabajo presentado.

Públicamente agradecemos al personal docente de la carrera de Diseño Gráfico de la Universidad Nacional de Chimborazo: Ph.D. Adalberto Fernández Sotelo, MSc. Marcela Cadena, MSc. Mariela Samaniego, MSc. Elvis Ruiz; un especial reconocimiento al MSc. Jorge Ibarra L. dilecto colega investigador que aportó significativamente en el desarrollo de los ajustes metodológicos y confección de las rúbricas de evaluación para los PIS.

Así también, agradecemos al personal docente y estudiantes de la carrera de Comunicación Social, mención Publicidad y Diseño Gráfico de la Universidad Internacional del Ecuador-sede Guayaquil: Mgs. Dennys Jordán C., Mgs. María Verónica Maldonado y Lic. Andrea Zunino. Un reconocimiento a los estudiantes líderes del proyecto: Amy Intriago y Luis Ortega; en su persona a todos los estudiantes que participaron en el Día de Simón.

\section{ANEXO 1.- Rúbricas de evaluación para Proyectos Integradores de Saberes UIDE/UNACH}




\section{Rúbrica De Evaluación Pis "Día De Simón” Uide}

Se calificará sobre categorías:

\section{Sobresaliente (5), Muy bueno (4), Bueno (3), Regular (2), Insuficiente (1)}

\begin{tabular}{|c|c|c|c|c|c|c|}
\hline \multirow[t]{2}{*}{$\mathbf{N}^{\mathbf{o}}$} & \multirow[t]{2}{*}{ INDICADORES } & \multicolumn{5}{|c|}{ VALORACIÓN } \\
\hline & & 5 & 4 & 3 & 2 & $\mathbf{1}$ \\
\hline 1 & Identifica el problema de manera correcta & $\mathrm{x}$ & & & & \\
\hline 2 & Formula los objetivos del proyecto & & $\mathrm{x}$ & & & \\
\hline 3 & $\begin{array}{c}\text { Diseña instrumentos de recolección de } \\
\text { información }\end{array}$ & $\mathrm{x}$ & & & & \\
\hline 4 & $\begin{array}{c}\text { Sistematiza con claridad los resultados de } \\
\text { investigación }\end{array}$ & $\mathrm{x}$ & & & & \\
\hline 5 & Emplea los contenidos tratados & $\mathrm{x}$ & & & & \\
\hline 6 & Es tolerante con la opinión de los demás & & $\mathrm{x}$ & & & \\
\hline 7 & $\begin{array}{l}\text { Asiste puntualmente a las reuniones } \\
\text { planificadas }\end{array}$ & $\mathrm{x}$ & & & & \\
\hline 8 & Coordina el trabajo en equipo & & $\mathrm{x}$ & & & \\
\hline 9 & Expresa con claridad sus opiniones & & $\mathrm{x}$ & & & \\
\hline 10 & $\begin{array}{c}\text { Demuestra solvencia y confianza al expresar } \\
\text { sus conocimientos, presentando la información } \\
\text { más precisa y pertinente para el desarrollo del } \\
\text { tema. }\end{array}$ & $\mathrm{x}$ & & & & \\
\hline 11 & $\begin{array}{l}\text { Ofrece una exposición altamente organizada, } \\
\text { respetando los tiempos establecidos, facilitando } \\
\text { la captación de su explicación desde el inicio } \\
\text { hasta el final de su intervención. }\end{array}$ & $\mathrm{x}$ & & & & \\
\hline 12 & $\begin{array}{l}\text { Establece un permanente contacto con el } \\
\text { público a través del dominio de un registro } \\
\text { lingüístico adecuado, un buen tono de voz, el } \\
\text { código gestual y el contacto visual. }\end{array}$ & $\mathrm{x}$ & & & & \\
\hline
\end{tabular}

\section{Rúbrica De Evaluación Pis “Manual De Identidad De Marca” Unach}



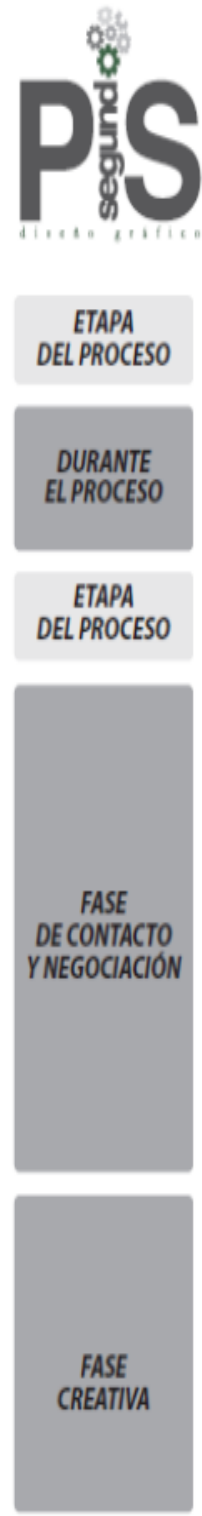
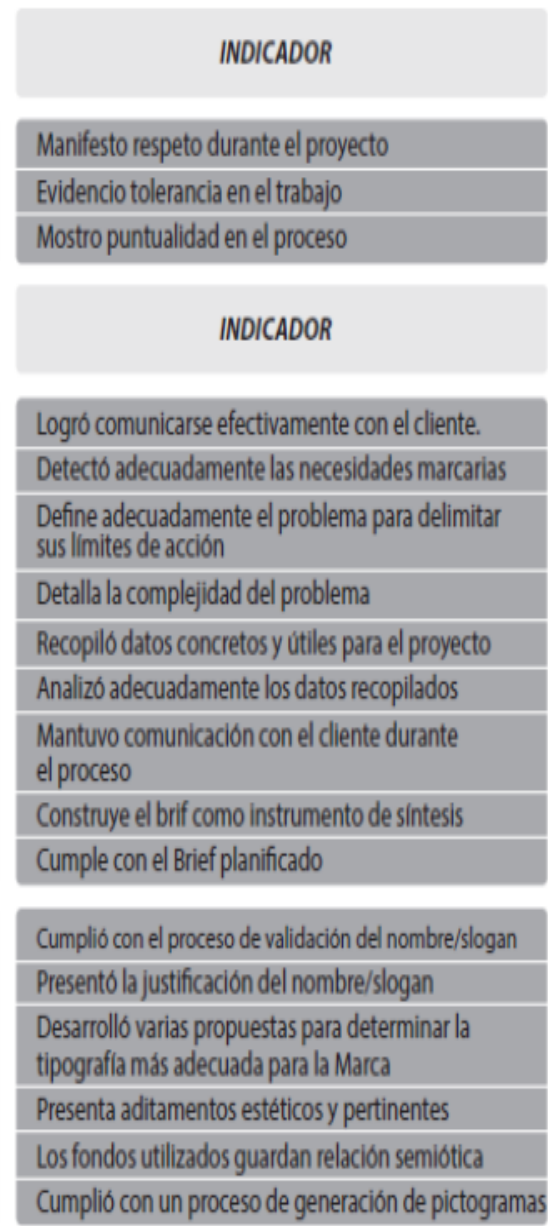

UNTVERSIDAD NACIONAL DE CHIMBORAZO

FACULTAD DE CIENCLAS DE LA EDUCACIÓN HUMANAS Y TECNOLOGÍAS

CARRERA DE DISEÑO GRÁFICO

La presente rúbrica evalua el proyecto desarrollado por el grupo:

Fecha:
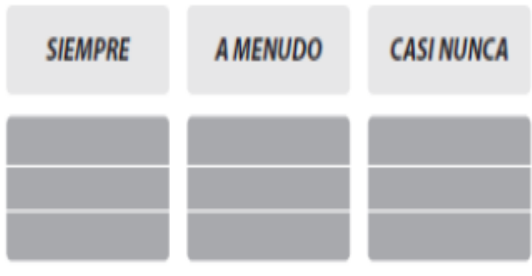

NUNCA
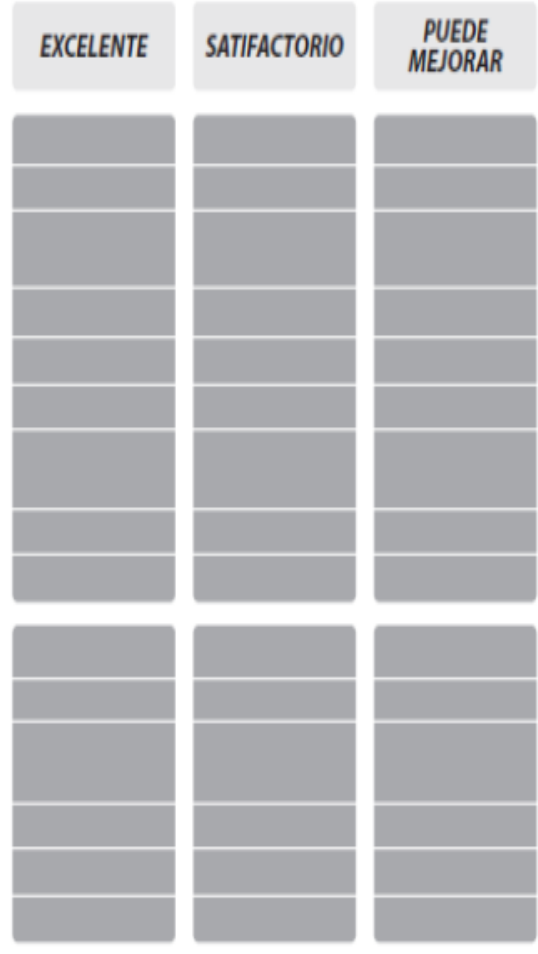

INADECUADO
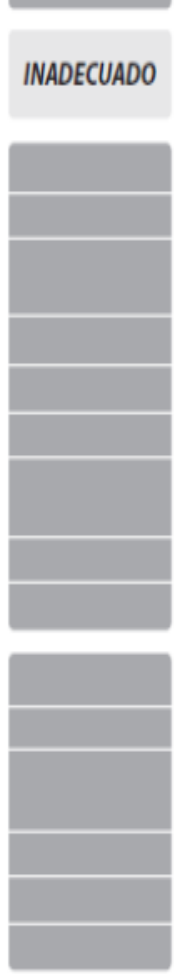

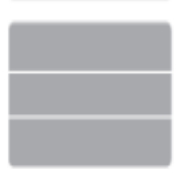




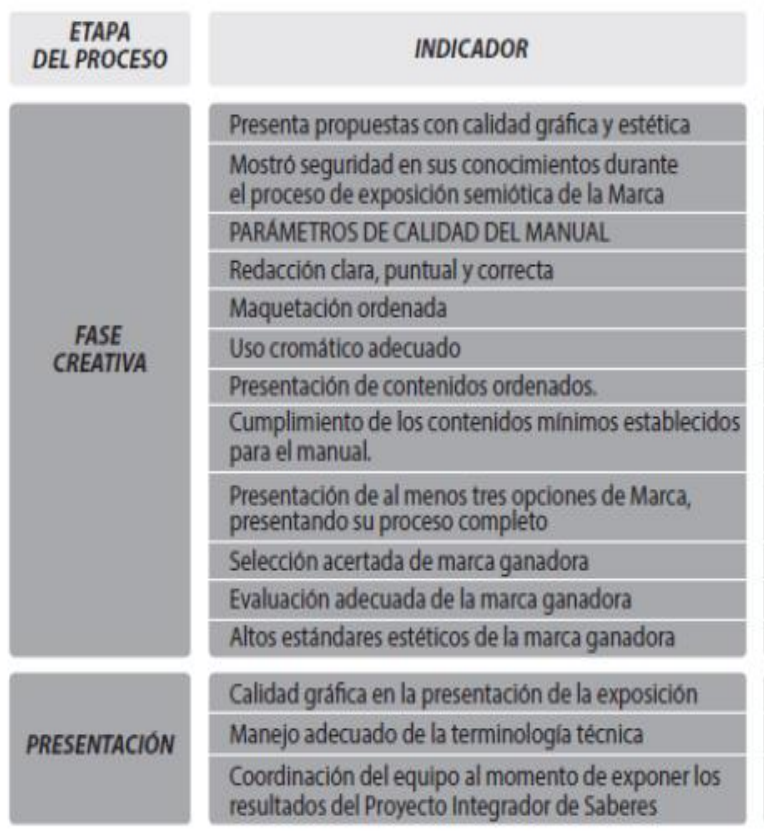

Puntaje por nivel logrado en un $100 \%$

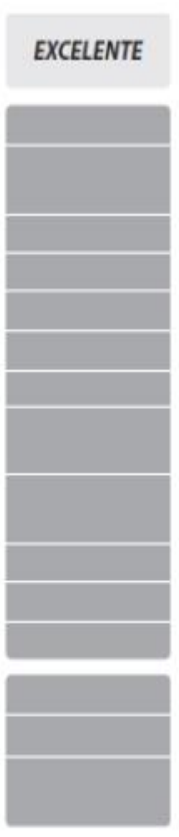

10

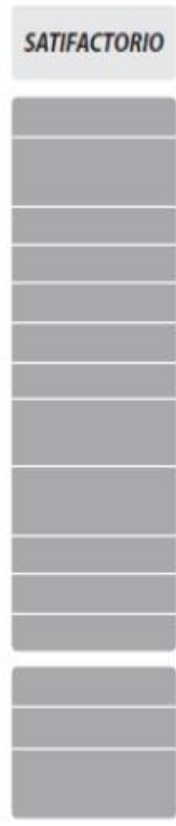

$9 \cdot 8$

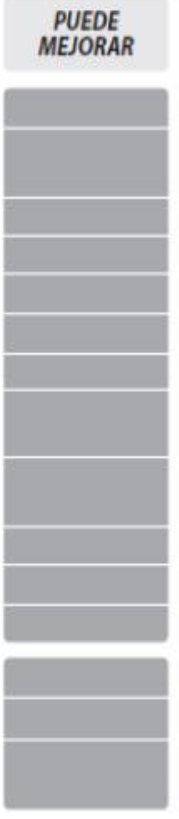

$7 \cdot 5$
INADECUADO

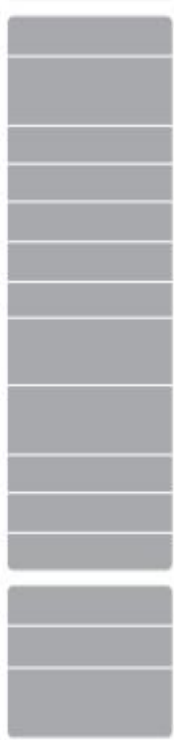

5-2

Observaciones:

Puntaje obtenido por PIS

NOTA FINAL PIS 